\title{
La constitución del programa de Hilbert*
}

The Constitution of Hilbert's Program

Max Fernández de Castro ${ }^{\dagger}$

Yolanda Torres Falcón ${ }^{\ddagger}$

\begin{abstract}
Resumen
En las páginas que siguen pretendemos dar una visión panorámica y esquemática de la evolución del programa formalista que resulta de los estudios recientes de notas de curso hasta hace poco inéditas. Analizaremos primeramente ciertos elementos del programa (la preferencia por el método axiomático, el estructuralismo y el logicismo). En segundo lugar observaremos cómo, una vez el programa establecido en 1920 (aunque con cierta vaguedad), diversos finitismos con una base común fueron ensayados por Hilbert y Bernays hasta 1931, en una tentativa por definir con precisión su programa y llevarlo a buen término. El resultado es el de un complejo programa de investigación en continua evolución.
\end{abstract}

Palabras clave: Hilbert - Bernays - logicismo - axiomático - finitismo

\begin{abstract}
In the pages that follow, it is our intention to present a panoramic and schematic view of the evolution of the formalist program, which derives from recent studies of lecture notes that were unknown until very recently. Firstly, we analyze certain elements of the program (the preference for the axiomatic method, as well as structuralism and logicism). Secondly, we observe how, once the program was established in 1920 (albeit somewhat vaguely), in the period up to 1931, different types of finitism with a common basis were tried out by Hilbert and Bernays, in an effort to define their program precisely and bring it successfully to fruition. The result is a complex research program in constant evolution.
\end{abstract}

Keywords: Hilbert - Bernays - logicism - axiomatics - finitism

* Recibido: 11 de abril de 2018. Aceptado con revisiones: 7 de mayo de 2018.

† Departamento de Filosofía, Universidad Autónoma Metropolitana-Iztapalapa, México. Para contactar al autor, por favor, escribir a: xamf_mx@yahoo.com.

$\$$ Departamento de Filosofía, Universidad Autónoma Metropolitana-Iztapalapa, México. Para contactar al autor, por favor, escribir a: yolatorresfalcon@gmail.com.

Metatheoria 10(2)(2020): 31-50. ISSN 1853-2322. eISSN 1853-2330.

(C) Editorial de la Universidad Nacional de Tres de Febrero.

(C) Editorial de la Universidad Nacional de Quilmes.

Publicado en la República Argentina. 


\section{La prehistoria del programa de Hilbert y algunos de sus elementos constitutivos}

Una primera lectura de los escritos publicados por Hilbert en torno a las matemáticas puede dejar la impresión de que las ideas centrales de su programa formalista aparecen, aunque con cierta vaguedad, desde comienzos del siglo XX. En particular se encuentra en su correspondencia con Frege la idea de que la consistencia de un sistema axiomático es todo lo que el matemático requiere para validar la existencia de los objetos o conceptos de su ciencia. Asimismo, la idea de que una prueba de consistencia absoluta de una disciplina matemática puede conseguirse formalizándola y tomando como nuevos objetos de estudios los símbolos de esta formalización, junto con sus reglas sintácticas, aparece esbozada en una conferencia de 1904. Sin embargo, la impresión de que el programa formalista se desarrolla a partir de allí de una manera lineal, con sólo un incremento en las precisión de los temas que lo constituyen, puede ser refutada por los mismos textos publicados de Hilbert. Más aún, el estudio de las notas de cursos que impartió sobre los fundamentos de las matemáticas, primeramente solo y, a partir de 1917, en colaboración con Bernays, hasta 1920, produce una impresión muy diferente, a saber, la de una oscilación entre posturas muy variadas: logicismo, finitismo muy estricto y un cierto formalismo metodológico. A partir de esa fecha el programa parece mejor definido, excepto por la cuestión de los medios aceptables para una prueba de consistencia, que sigue siendo tema de experimentación. Es lo que pretendemos mostrar en las páginas que siguen, trazando un panorama esquemático de evolución del pensamiento de Hilbert en torno a los fundamentos de las matemáticas en las primeras tres décadas del siglo XX. Comenzaremos por analizar ciertos elementos constitutivos del programa o muy relacionados con él.

\subsection{El método axiomático}

Para empezar repasemos algunos rasgos de la axiomatización de la geometría realizada por Hilbert en 1899. Como es sabido, uno de los puntos de su disputa con Frege es que para éste hay una separación tajante entre axiomas y definiciones. Tradicionalmente los axiomas eran entendidos como aserciones respecto a conceptos y objetos identificados previamente (tal vez por definiciones o intuitivamente). En Los fundamentos de la Geometría de Hilbert (1899) culmina una nueva concepción del método axiomático que venía fraguándose en el siglo XIX y según la cual los axiomas constituyen definiciones implícitas de los términos que en ellos aparecen. Para comparar estas concepciones conviene cotejar la manera en que justifican ciertos rasgos que ambas atribuyen al método axiomático. Uno de estos rasgos es que los axiomas deben ser lógicamente independientes (preocupación presente en la axiomática de Euclides, como lo muestran los intentos de prueba del quinto postulado). Un axioma dependiente sería redundante desde el punto de vista de la inferencia (método antiguo) o desde el punto de vista de la determinación de los conceptos u objetos que son definidos por los axiomas (método moderno). Otro rasgo es que las pruebas no pueden recurrir a ninguna propiedad de los conceptos u objetos de la disciplina en cuestión salvo los que han sido explícitamente señalados en los axiomas. Esta exigencia es parte constitutiva de la versión moderna, mientras que en la antigua corresponde a la idea de que los axiomas encapsulan lo que es esencial al tema de estudio. El tercer atributo es que los axiomas deben ser completos, no en los sentidos de la lógica contemporánea, sino en el de ser suficientes para la demostración de todas las verdades que se espera sean demostrables en esa disciplina. En la concepción antigua corresponde al mencionado deseo de comprimir en los axiomas toda la materia en cuestión. En la axiomática moderna parecería una exigencia huera, pues si los axiomas definen implícitamente a los objetos de que tratan ¿cómo podría estos tener propiedades que no fuesen consecuencia lógica de los axiomas? Una posibilidad es que normalmente la axiomatización se propone como la formalización de una disciplina que se ha desarrollado de otra manera y, aunque los objetos o conceptos definidos por los axiomas no son necesariamente los mismos de la teoría original, sí deben corresponder a ellos en cierto sentido. Por ello la axiomatización debe recuperar completamente los teoremas primigenios. 
Otra posibilidad, presente ya en Dedekind y, más tarde, en Huntington, es que la completud se refiera a la categoricidad: todos los modelos son isomorfos y, por lo tanto, los axiomas determinan un único modelo (salvo isomorfismo). Si no fuese así, podrían plantearse preguntas que no pudieran decidirse a partir de los axiomas. Es decir los conceptos u objetos definidos no satisfarían la ley del tercero excluido. Una última propiedad exigida a los axiomas es que formen un conjunto consistente. En la versión tradicional esta es una propiedad que se sigue de que los axiomas son aserciones verdaderas y que la inferencia lógica transmite la verdad de las premisas a la conclusión. En cambio en la axiomática moderna es un requisito esencial. Los axiomas no definirían nada si fuesen contradictorios. Para Hilbert 'consistencia' significa la imposibilidad de deducir una contradicción en un número finito de pasos y había sido probada para diversos sistemas en su obra de 1899 vía la exhibición de modelos: al asignar significado a los términos no lógicos de un sistema axiomático para la geometría, los axiomas devienen verdades de alguna otra disciplina, por ejemplo, del análisis, lo que muestra que la existencia de una contradicción en la geometría implicaría la existencia de una contradicción en el análisis. Eran pruebas de consistencia relativa.

Frege entiende el método axiomático en el sentido tradicional. Sin embargo, admite que un conjunto de axiomas puede ser considerado como una definición siempre que aceptemos que lo que define es una relación de orden superior (entre conceptos de orden menor, o entre conceptos y objetos). Hilbert concuerda con Dedekind (aunque también con Poincaré) en que en matemáticas la consistencia implica existencia. Más precisamente, la existencia matemática se reduce a la consistencia. Pero Si los axiomas forman un conjunto consistente, ¿a qué debemos atribuir existencia? $\mathrm{O}$, más precisamente, ¿qué es lo que los axiomas definen? Partamos de un ejemplo. Por simplicidad supongamos un sistema cuyos únicos axiomas son:

Una línea pasa a través de cualesquiera dos puntos.

Cualquier línea pasa por al menos tres puntos.

Y que esos axiomas definen 'punto', 'línea' y 'pasa a través' como una relación entre líneas y puntos. En notación moderna la conjunción de esos axiomas puede ser expresada por la fórmula:

$$
\begin{aligned}
& {[(\forall x)(\forall y)((x \neq y \wedge P x \wedge P y) \rightarrow(\exists z)(L z \wedge P z x \wedge P z y)]} \\
& \wedge(\forall x)(L x \rightarrow(\exists y)(\exists z)(\exists w)(y \neq z \wedge z \neq w \wedge y \neq w \wedge P x y \wedge P x z \wedge P x w)
\end{aligned}
$$

Si consideramos, además, que nada más es presupuesto (con respecto al significado de los términos extralógicos), entonces 'P', 'L' y 'C' en la fórmula figuran como variables libres y la fórmula define una relación $\mathrm{T}$ que resulta entre pares de conceptos de primer orden y una relación binaria (también de primer orden). La extensión de ' $\mathrm{T}$ ' es de la forma:

$$
\{(\mathrm{A}, \mathrm{B}, \mathrm{R}),(\mathrm{C}, \mathrm{D}, \mathrm{R}),(\mathrm{M}, \mathrm{N}, \mathrm{Q}) \ldots\}
$$

Para Frege estarán involucrados la relación de segundo orden $\mathrm{T}$, los conceptos A, B, R, C, etc., un concepto de segundo orden bajo el que caen A, C, M..., y por últimos los objetos subsumidos por estos conceptos. Ahora bien, ¿qué es lo que los axiomas definen, si los axiomas son consistentes? Desde el punto de vista fregeano no habría más que las opciones indicadas. En cambio Hilbert llamaría a los miembros de $\mathrm{A}$, a los de $\mathrm{C}$ y a los de $\mathrm{M}$ "puntos". Los axiomas definirían un concepto de primer orden 'punto' pero, nótese, no un concepto fregeano. Hilbert diría que un elemento de A es un punto sólo con relación al sistema (A, B, R). Su adversario le recrimina que la definición axiomática no le permite determinar si su reloj de bolsillo es un punto. En la concepción fregeana, una vez definido el concepto, tiene sentido preguntar de cualquier objeto si es un punto o no. Para Hilbert, al contrario, la pregunta aislada de si Julio César es el número 2 no tiene ningún sentido. Sólo lo tiene con relación a una cierta red de conceptos que cumplen las condiciones establecidas en los axiomas. En este caso, Hilbert estaría proponiendo una concepción novedosa de los conceptos y a ello aludiría la frase final de su correspondencia con Frege (que citaremos más adelante). Si es así, hay una diferencia radical entre ambos antagonistas. 
Hilbert (1899) llama al método axiomático antiguo material, y al nuevo, "formal". A esta manera de construir o determinar un domino de objetos matemáticos la contrapone con el método genético. Un ejemplo de uso del método genético es la constitución paso a paso de los números naturales desde el 1 a su sucesor y de éste al 3, etc., por las necesidades de la operación de contar y la posterior ampliación del conjunto de números así obtenido al domino de los enteros, para eliminar restricciones en el proceso de la substracción y, ulteriormente, continuar con otras ampliaciones sucesivas hasta llegar al campo de los números reales o de los complejos. Podría pensarse que Hilbert defendió el método axiomático por el papel que éste juega en la realización de pruebas de consistencia (ya en el programa formalista). No es así, para él, el método axiomático es el adecuado para capturar o determinar los objetos de estudio de las matemáticas. Así dice, por ejemplo:

De acuerdo con lo dicho, por conjunto de los números reales no tenemos que entender la totalidad de las leyes posibles según las cuales pueden avanzar los elementos de una sucesión fundamental, sino más bien, como acabamos de decir, un sistema de objetos cuyas relaciones se encuentran determinadas por el sistema finito y cerrado de los axioma I-IV, y en relación al cual ninguna afirmación será válida si no puede deducirse a partir de esos axiomas por medio de un número finito de inferencias lógicas (Hilbert 1993, p 21).

Hilbert insiste en que las cortaduras de Dedekind pueden servir para determinar números reales particulares, pero no sirven como definición de lo que es un número real. ¿Por qué? Porque una cortadura particular es un número real sólo dentro de una red conceptual dada por un sistema axiomático.

\subsection{El estructuralismo}

Se ha señalado repetidamente que Hilbert es un precursor del estructuralismo en filosofía de las matemáticas y que en esto también siguió a Dedekind. Es claro que la idea de que el matemático estudia estructuras va de la mano con el desarrollo de axiomática moderna. Shapiro (1997) distingue tres clases de estructuralismo. El primero, ante rem, es de linaje platónico: las estructuras existen y son independientes de sus ejemplificaciones concretas. Para el segundo, in re, de corte aristotélico, el matemático estudia estructuras que son comunes a varios sistemas, pero sin suponer que esas estructuras son algo diferente de los sistemas que las ejemplifican. El tercero, el modal, es similar al anterior excepto que los sistemas en cuestión no necesitan ser reales, basta con que sean posibles. Los últimos dos son llamados "eliminativos". A partir de la evidencia textual es difícil atribuir a Hilbert alguna de estas posiciones. Un párrafo de Bernays muestra esta ambigüedad:

La estructura lógica de la geometría axiomática en el sentido de Hilbert [...] es puramente hipotética. Si en la realidad hay, de alguna manera, tres sistemas de objetos, así como relaciones determinadas entre esos objetos, tales que los axiomas de la geometría sean válidos respecto a ellos [...] entonces todos los teoremas de la geometría son válidos para esos objetos y también para esas relaciones. Así el sistema axiomático mismo no expresa nada factual; más bien, presenta sólo una forma posible de un sistema de conexiones que debe ser investigado matemáticamente de acuerdo a sus propiedades internas. (Bernays 1922, en Mancosu 1998, p. 122)

Tal vez sería correcto decir que Hilbert es indiferente a las distinciones ontológicas planteadas por Shapiro.

Frege criticó a Hilbert por demostrar la consistencia lógica de un conjunto de axiomas a través de la existencia de ciertos modelos, siendo que Hilbert parece definir la existencia en términos de consistencia. Evidentemente la objeción era correcta hasta 1904. Las pruebas de consistencia dadas hasta entonces sólo trasladaban el problema de la consistencia de un sistema axiomático al de la consistencia de otro. Sin embargo, si Hilbert estuviese defendiendo una forma de estructuralismo eliminativo, podría decir que la existencia de un modelo, a la vez que demuestra la no contradictoriedad de los axiomas (supuesta la consistencia de otro sistema), prueba la existencia de la estructura en cuestión, pues ésta sólo existe como parte del sistema que la ejemplifica. 


\section{3. ¿Logicismo?}

Sieg $^{1}$ (2013) y Ferreirós (2009) han señalado que Hilbert profesó un forma de logicismo, tal vez de forma intermitente, en un periodo que va desde la última década del siglo XIX hasta 1920. Esta fecha final es indudable. Menos claro es si esta tendencia logicista fue una constante que sólo sufrió un breve eclipse en torno a 1904 o fue una tendencia que surgió y desapareció varias veces a lo largo de ese periodo. Ferreirós se inclina por lo primero y sus argumentos hacen plausible su tesis, pero no podemos pasar aquí de un nivel especulativo. Sin embargo, hay que proceder con cuidado. ¿Qué le atribuimos a Hilbert al llamarlo "logicista"? La primera respuesta es: muy poco. El logicismo puede ser entendido de varias maneras. Su núcleo básico está compuesto por dos tesis, una conceptual y otra doctrinal. La primera es que lo conceptos matemáticos pueden ser definidos exclusivamente en términos de conceptos lógicos. La segunda es que, una vez hecha esta reducción conceptual, los teoremas matemáticos pueden demostrarse únicamente a partir de los principios de la lógica. Adviértase cómo el logicismo, así entendido, es compatible con filosofías de la matemática muy diversas, y puede incluso servirles de apoyo. No es difícil imaginar un logicismo kantiano, uno antikantiano (como fue el de Frege), uno empirista (como el de los miembros del Círculo De Viena). En este sentido, el logicismo no es todavía una filosofía de la matemática. ${ }^{2}$

Estrictamente el logicismo de Dedekind y Frege sólo se extiende a la aritmética, lo que incluye todo el análisis y el álgebra pero no, por supuesto, la geometría. El siguiente pasaje pudo ser de la autoría de cualquiera de ellos:

La Geometría es el estudio de las propiedades del espacio. Es esencialmente diferente de los dominios puramente matemáticos del pensamiento, verbigracia, la teoría de los números, el álgebra, la teoría de funciones. Los resultados de esos dominios pueden ser obtenidos por el pensamiento puro en [el sentido en] que uno reduce los hechos aseverados a otros más simples, a través de claras inferencias lógicas, hasta que al final uno sólo necesita el concepto de número entero [...] y uno puede obtener el concepto de número entero también por el pensamiento puro”. (Citado en Ferreirós 2009, p. 36)

Ese párrafo fue escrito por Hilbert en 1891.

¿En qué consistía la convicción logicista de Dedekind? Estaba convencido de que los conceptos y operaciones aritméticas se componen de operaciones y conceptos sin los cuales el entendimiento humano no puede proceder. La fe logicista de Frege se basaba en que parece imposible concebir falsas las leyes aritméticas más simples "sin que todo caiga en la confusión" (Frege 1953, párrafo 14). Pero, además de esta profesión de fe en el par de tesis mencionadas, el logicismo fue un programa reduccionista. Tanto Dedekind como Frege se propusieron probar las dos tesis mencionadas y transformaron al logicismo, cada cual a su modo, en un programa matemático. La cita que dimos anteriormente muestra que Hilbert profesaba el logicismo en el sentido mínimo que mencionamos, pero ¿podemos encontrar en su obra temprana una contribución al aspecto programático del mismo? Aparentemente podríamos articular la respuesta de la siguiente manera. Siguiendo a Ferreirós (2011) y a Benis-Sinaceur (2008), damos por bien establecida la influencia de Dedekind en el pensamiento y la práctica matemática de Hilbert. Podemos suponer que Hilbert aceptaba la reducción de la aritmética ya realizada por Dedekind en Zahlen cuando escribió el párrafo que acabamos de citar. Al parecer, Hilbert admitía incluso la prueba de la existencia de un conjunto infinito dada en el párrafo 66 de esa obra, al menos hasta 1899, y sólo la rechazó en sus escritos a partir de 1905. De acuerdo a Ferreirós (2009), las paradojas habrían despertado a Hilbert de su sueño dogmático, no al grado de hacerlo renunciar a su logicismo (por lo menos hasta 1904), pero sí lo habrían obligado a revisarlo en su versión dedekindeana. Habría entonces insistido en una prueba de consistencia más satisfactoria. Habría pasado de una concepción naïve de conjunto, a saber, la concepción lógica, o dicotómica, en que un conjunto existe si y sólo si hay un criterio para separar en dos los objetos del universo, a una

\footnotetext{
1 “Around 1900, [Hilbert] pursued, a kind of Dedekindian logicism and, almost 20 years later, he took quite seriously Russell's attempt of founding mathematics in logic" (Sieg 2013, p. 31).

2 Benis-Sinaceur (2015) argumenta que, dadas las diferencias entre los pensamientos y los programas de Frege y el de Dedekind, resulta engañoso clasificar a ambos como logicistas.
} 
concepción más compleja en que una condición para la existencia de un conjunto es que el sistema axiomático que lo define sea consistente. Los argumentos que cita Ferreirós son convincentes, pero hay que hacer algunas aclaraciones. Como él mismo señala, hay tres momentos sucesivos en que las paradojas irrumpen en la escena. En 1897, Cantor comunica a Hilbert ciertas antinomias de la naciente teoría de conjuntos. Un año o dos más tarde, Hilbert constata su importancia al reproducirlas en un marco dedekindeano. Finalmente en 1903, un poco después de que Russell comunicara a Frege su paradoja y al ver la alarmada reacción de éste, el círculo matemático de Göttingen les prestó mayor atención. Este último episodio podría haber dado pie a la renuncia temporal de Hilbert al logicismo, manifiesta en la conferencia de 1904. Sin embargo, no es tan fácil situar el punto en que Hilbert abandona la concepción dicotómica de "conjunto" y al logicismo dedekindeano. No es fácil por dos razones. La primera es que en algunos párrafos posteriores (por ejemplo, uno de 1905) ${ }^{3}$ Hilbert parece defender la concepción dicotómica. Otro es que Hilbert sólo rechaza explícitamente la prueba del párrafo 66 de Zahlen en 1904. Sin embargo, estos datos pueden acomodarse con la interpretación dada. Si Hilbert constató que las paradojas podían ser reproducidas en un marco dedekindeano y en 1900 consideró la prueba de la consistencia de aritmética como uno de los problemas centrales del siglo que empezaba, cabe conjeturar que ya no admitía la referida prueba de la existencia de un conjunto infinito. Por otro lado es claro que no admitía la concepción dicotómica de "conjunto" en la época de su correspondencia con Frege. En ella aparece el siguiente párrafo:

Tu ejemplo al final del libro [la paradoja de Russell] era conocido por nosotros aquí. Yo encontré otras contradicciones aún más convincentes hace 4 o 5 años: ellas me llevaron a la convicción de que la lógica tradicional es inadecuada y que la teoría de formación de conceptos necesita ser afilada y refinada. Como yo lo veo, la más importante falla en la estructura tradicional de la lógica es la presunción hecha por todos los lógicos y matemáticos hasta ahora de que un concepto está ya ahí si uno puede establecer de cualquier objeto si cae bajo él o no. Esto me parece inadecuado. Lo que es decisivo es el reconocimiento de que los axiomas que definen el concepto estén libres de contradicción. (Frege 1980, pp. 51-52)

Este último párrafo fue escrito en 1903, pero dice "hace 4 ó 5 años", lo que concuerda con la interpretación dada. Aquí podría surgir un malentendido. No hemos dicho que Dedekind no concediera importancia a la prueba de consistencia de un sistema axiomático o que aceptara la concepción dicotómica de conjunto. Lo que dijimos es que, ante la aparición de las paradojas y la conciencia de su importancia, Hilbert habría rechazado la prueba de consistencia dada en el párrafo 66 de "Was sind und was sollen die Zahlen" y habría renunciado a la concepción dicotómica de conjunto. ¿Cómo acomodar entonces el párrafo de 1905 en que parece defender la concepción dicotómica? Puede simplemente ser entendido como refiriéndose a conjuntos consistentes (para usar la terminología de Cantor). Eso pudo haber estado implícito en el escrito.

Como veremos, Hilbert abandonó definitivamente el logicismo a partir de 1920. Sin embargo, no debemos olvidar que hay un componente logicista en su programa formalista: no la tesis de la reducción de las matemáticas a la lógica, pero sí la convicción de que las formalizaciones de la lógica logradas hasta entonces por Frege o por Russell era "completas", en el sentido de abarcar todo el razonamiento lógico informal usado en el desarrollo de las matemáticas. Una formalización insuficiente de los principios lógicos no serviría al programa. La idea era que una prueba de consistencia de una teoría formalizada garantizaría la consistencia de la teoría informal correspondiente, y esto no podría conseguirse con una lógica incompleta. ${ }^{4}$

\subsection{4}

\footnotetext{
${ }^{3}$ Sobre el principio de comprensión dice Hilbert que es "el principio fundamental de que un concepto (conjunto) está definido y es inmediatamente utilizable si sólo está determinado para cada objeto si el objeto es subsumido bajo el concepto o no" (citado en Ferreirós 2009, p. 57).

${ }^{4} \mathrm{Al}$ respecto ver, por ejemplo, los comentarios de Hilbert al problema IV (Hilbert 1928).
} 
Como hemos dicho, la conferencia de 1904 (publicada en 1905) con el título "Über die Grundlagen der Logik und der Arithmetik", marca una ruptura en el pensamiento de Hilbert. En ella presenta un método novedoso para probar consistencia de un sistema de axiomas que anticipa algunas de las ideas centrales del programa formalista. Una es que las axiomatizaciones de disciplinas matemáticas deben incluir los principios y reglas de inferencia lógicas de que se sirvan en su desarrollo, lo cual supone, por supuesto, un abandono del logicismo. Otra es que esos sistemas pueden ser considerados como conjuntos de expresiones y de reglas que permiten generar teoremas a partir de las expresiones señaladas como axiomas. De esa manera las pruebas de un tal sistema axiomático pueden ser objeto de estudio matemático. Una prueba combinatoria puede demostrar que algunas fórmulas no podrán generarse como teoremas, lo que equivaldría a demostrar la consistencia del sistema en cuestión. Allí Hilbert critica por primera vez la prueba dedekindeana de la existencia de un conjunto infinito y sugiere que el concepto de "conjunto" no pertenece a la lógica. Como ejemplo de su método, Hilbert ofrece una prueba directa de la consistencia de un reducido sistema axiomático, considerando sólo sus rasgos sintácticos. Utiliza para ello la inducción matemática pues demuestra que si en una prueba de $n$ pasos no se ha generado una contradicción tampoco ocurrirá en una prueba con un paso extra. Como señalan Mancosu (1998, p, 153) y Ferreirós (2009, p. 64), las ideas de la conferencia de Heidelberg de 1904 distan mucho del programa de Hilbert aún en su primera versión, aunque lo anticipen en algunos puntos, pues no está allí delimitada con claridad la frontera entre matemática y metamatemática, y el marco lógico del sistema en cuestión sólo está esbozado. Además, aunque es obvio que la prueba metamatemática de consistencia debe recurrir sólo a medios epistémicamente seguros y que debe ser de tipo combinatorio, no hay mayor especificación de los mismos, ni alusión alguna al carácter finitario de la prueba.

A pesar de estas imprecisiones, Poincaré (1905-1906) encuentra una dificultad en el ejemplo con que Hilbert ilustra su método e intuye con acierto que será un obstáculo a la solución definitiva del problema de la consistencia, a saber, que la prueba recurrirá al principio de inducción matemática. La objeción toma dos formas. En 1905 el problema radica en una forma de circularidad: la prueba supondrá la validez de la aritmética (o del principio de inducción) para demostrar su propia consistencia. Después de que en la discusión con Russell aparece el concepto de impredicatividad y el diagnóstico de que en él se encuentra las raíz de las paradojas, Poincaré objeta a Hilbert que, o bien la prueba recurrirá a un principio más poderoso que el de inducción, o bien echará mano de un definición impredicativa. Volveremos a este tema más adelante.

\section{5. $1905-1917$}

Muy pronto Hilbert encuentra insatisfactoria su propuesta presentada en Heidelberg y comienza un estudio más serio de los principios lógicos. Sin embargo, de acuerdo a Sieg (1981, pp. 97-101), pocas cosas dignas de atención referentes a nuestro tema hay en las notas correspondientes al periodo que va de 1905 al verano de 1917. En las de 1910 se dice que la paradoja de Richard es una falacia. Ahí observa que los signos lingüísticos pueden ser sometidos a operaciones cuyas reglas son similares a las de la aritmética. En las del verano de 1917, se encuentran algunas observaciones interesantes. Una es que la paradoja de Russell, aunque eliminada del análisis por la axiomatización de Zermelo, no ha sido resuelta satisfactoriamente. Hilbert relaciona este problema a lo que llama definiciones "genéticas", las cuales incluyen todas las impredicativas. Adopta entonces un punto de vista novedoso que proscribe el uso de expresiones como 'todo', 'cada' y así sucesivamente'. Aquí aparece, tal vez de manera sólo tentativa y exploratoria, una forma extrema de finitismo (que, de acuerdo a Hallet, ya había sido esbozada en 1904-5):

No hay necesidad de considerar a los números irracionales; La serie geométrica $1+1 / 2+1 / 4+1 / 8$ "y así sucesivamente" es ya un ejemplo. Ni siquiera fórmulas en las cuales figuran números enteros finitos $n$, pero sólo indeterminados, son inmunes a nuestra crítica. Para ser capaz de aplicarlas uno considera $n=1$, 2, 3, 4 "y así sucesivamente". Kronecker, quien intentó reducir todas las matemáticas a los números enteros, no fue suficientemente radical, pues ' $n$ ' figura en su fórmula. Debería haberse limitado a los 
números específicos 7, 15, 24. Así, uno ve qué tipo de dificultades tendrían que ser enfrentadas al calcular con letras. La simple fórmula $\mathrm{a}+\mathrm{b}=\mathrm{b}+\mathrm{a}$ puede ya ser atacada (citado en Sieg 1981, pp. 99-100).

Es esta la caracterización de un finitismo mucho más radical que el de Kronecker. Las variables están proscritas, tal vez porque conllevan implícitas las referencias a un cuantificador universal. Lo curioso que esta cita aparece junto a otras que favorecen una forma de logicismo. Por ejemplo, en respuesta a Poincaré, Hilbert dice que una ulterior reducción de los axiomas de la aritmética es posible, pero que eso será investigado en el siguiente trimestre. No es claro cómo se combinaría ese finitismo extremo con un logicismo. Veremos que el ejemplo de la fórmula ' $\mathrm{a}+\mathrm{b}=\mathrm{b}+\mathrm{a}$ ' reaparece repetidas veces y que al menos desde 1922 hasta 1927 puede dársele una interpretación similar. Sin embargo, en 1917 dista mucho de ser claro su sentido.

En la conferencia de Zúrich dictada en noviembre de ese año, "El pensamiento axiomático", Hilbert reitera la importancia del método axiomático y su uso en diferentes ciencias. Uno de los requerimientos del método es que el conjunto de axiomas sea consistente. En el caso de las ciencias empíricas esto es debido a que "la existencia de contradicciones pone en entredicho la existencia de la teoría misma" (Hilbert 1993, p. 28). En el caso de las matemáticas no se trata de modificar la teoría conforme las contradicciones aparecen, sino de mostrar que éstas son imposibles. Enseguida alude a las pruebas de consistencia relativa como las que se encuentran en Grundlagen der Geometrie. Sin embargo, este método tiene un límite en los casos de la los números enteros y de la teoría de conjuntos. "Ahora bien, en vista del carácter ineludible de una demostración de consistencia, parecería necesario axiomatizar en primer lugar la lógica y probar luego que tanto la teoría de números como la de los conjuntos no son otra cosa que parte de ella" (Hilbert 1993, p. 31). Dice que este camino ha sido preparado por Frege y agrega "pero ha sido el agudo lógico y matemático B. Russell quien finalmente y con mayor éxito lo ha recorrido" (Hilbert 1993, p. 31). Es curioso que Hilbert no señale allí ningún problema en relación a la consecución del programa russelliano. Sólo dice que se plantea una nueva tarea que incluye, por ejemplo, investigar la decidibilidad de teorías. Tampoco hay allí ninguna alusión al finitismo esbozado en el curso anterior.

\section{La evolución del programa de Hilbert en su etapa madura}

\subsection{El nacimiento del programa formalista}

En ese mismo año Hilbert contrató a Bernays como su asistente en Göttingen y comenzó así una fructífera colaboración de muchos años. Al parecer los cursos subsiguientes fueron dedicados a investigaciones lógicas y, de acuerdo a Sieg, gran parte del material de los mismos es el que dio contenido al libro que Hilbert publicó en coautoría con Ackermann en 1928, Gründzüge der theoretischen Logik. Para lo que hace a nuestro tema importa señalar que las notas de las lecciones de invierno de 1917 contienen un desarrollo de la lógica de primer orden y una extensión ulterior al cálculo funcional. Hilbert y Bernays reconocen que las paradojas, como la de Russell, tienen su origen en la impredicatividad y, para evitarlas, adoptan la teoría de tipos. Por otro lado, para el desarrollo del análisis agregan el axioma de reducibilidad. Sin otra reserva, reconocen simplemente que este axioma entra en conflicto con el punto de vista constructivista de Principia Mathematica.

Esta situación perduró hasta 1920. En las notas de ese año encontramos una renuncia, esta vez definitiva, al logicismo. Finalmente la necesidad de aceptar el axioma de reducibilidad aparece como un defecto insuperable del logicismo russelliano:

Así, solo queda la posibilidad de asumir que el sistema de predicados y relaciones de primer orden es una totalidad existiendo independientemente y que satisface el axioma de reducibilidad. De este modo regresamos al punto de vista axiomático y renunciamos al objetivo de un fundamento lógico de la aritmética y el análisis. Porque ahora una reducción a la lógica sólo es dada nominalmente. (Citado en Sieg 2013, p. 108) 
Es decir, debemos suponer que hay un modelo de la lógica de primer orden con el axioma de reducibilidad. El problema de la consistencia no quedó entonces resuelto por la reducción logicista y, por lo tanto, no se había ganado nada. Era necesario buscar otra salida.

En estos párrafos hemos estado siguiendo los reportes de Sieg sobre las notas de curso de ese periodo. En el punto que ahora trataremos, también admitiremos su interpretación. De acuerdo a ésta, el programa de Hilbert, en su forma primigenia, surgió en tres etapas sucesivas, todas ellas reportadas en la notas de 1920. La primera, como vimos, es la renuncia al logicismo. La segunda, al parecer, es un intento de fundar la matemática en una forma muy radical de finitismo cuya motivación es "reconstruir la teoría de los números y moldear formas e inferencias de tal manera que las paradojas sean excluidas desde el principio y que los procedimientos de prueba se vuelvan inspeccionables" (citado en Sieg 2013, p. 112). Para esta forma de finitismo,

tal ecuación $\left[\mathrm{x}^{+} \mathrm{y}^{\mathrm{y}} \mathrm{y}^{+\mathrm{x}}\right]$ no es una aseveración para todos los números, sino que es interpretada de tal modo que su significado completo es dado por un procedimiento de prueba: cada paso del procedimiento es una acción que puede ser completamente exhibida y que sigue reglas fijas. (Citado en Sieg 2013, p. 114)

¿Por qué no es una aserción universal? Porque de ella, dice explícitamente Hilbert, la ecuación ' $2+3=3+2$ ' no es un caso especial y porque en la prueba "el concepto general de número no es todavía asumido". Aquí aparece una mención aprobatoria al rechazo del tercero excluido por parte de Brouwer.

El que el sentido de una aserción esté dado por una prueba hace pensar, en efecto, en el intucionismo. En la interpretación Brouwer-Heyting-Kolmogorov (BHK), la prueba de una aserción general es una instrucción para generar pruebas de casos particulares. Como dijimos, el ejemplo reaparecerá en las publicaciones subsiguientes de Hilbert sobre este tema, a veces con una mayor explicación de su sentido. Veremos que la interpretación BHK es compatible con estas explicaciones. Sin embargo, parece oponerse a ella la aserción de que la ecuación ' $2+3=3+2$ ' no es un caso especial de ' $\mathrm{x}^{+} \mathrm{y}=\mathrm{y}^{+} \mathrm{x}$ '. Sostendremos que Hilbert y Bernays hacen aquí una distinción entre la prueba de un enunciado universalmente cuantificado y un esquema de prueba para producir pruebas de instancias particulares.

Nótese que este finitismo, y tal vez lo mismo pueda decirse del esbozado en las notas de 1917, no forma parte de un programa para una prueba metamatemática de consistencia. Al parecer, estaría en la base de un proyecto fundacionista al nivel de la matemática misma. Esto debe sonar muy extraño al lector contemporáneo.

De acuerdo a la interpretación de Sieg, Hilbert constató rápidamente la imposibilidad de fundar la matemática sobre tan magra base y volvió a las ideas esbozadas en 1905. Con ello surge propiamente el programa formalista. Desvinculado de todo marco o justificación filosófica, el programa aplicado a una teoría matemática consiste, en primer lugar, en formalizarla axiomáticamente, incluida la lógica que emplea, de tal manera que sus pruebas se conviertan en meras sucesiones simbólicas regidas por reglas fijas de tipografía y, en segundo lugar, demostrar de manera conspicua que una fórmula determinada no podrá ser generada como teorema por esas reglas. Con ello quedará probada la consistencia del sistema en cuestión. Como ejemplo de la fecundidad del método, Hilbert esboza una prueba para una parte muy limitada (finitaria) de la aritmética elemental. El finitismo aparece aquí de nuevo a nivel matemático, pero no como fundamento, sino como una etapa preparatoria a la prueba de consistencia de la aritmética.

Nótese que la aritmética juega un doble papel en el programa. Por un lado, es la teoría cuya prueba de consistencia garantizaría que son absolutamente consistentes varias teorías para las que sólo había hasta entonces pruebas de consistencia relativa. Por otro lado, la prueba metamatemática de consistencia debe tratar el formalismo matemático como un sistema sintáctico, es decir, como un

\footnotetext{
${ }^{5}$ En particular, el capítulo "I.3 Hilbert's Programs: 1917-1922” de Sieg (2013).
} 
sistema aritmético. Esta equiparación entre sintaxis y aritmética había aparecido ya en las notas de Hilbert de 1910, como vimos anteriormente.

\title{
2.2. $1922-1927$
}

En las páginas que siguen revisaremos brevemente las sucesivas publicaciones de Hilbert en los años veintes en torno a los fundamentos de las matemáticas. Como dijimos, el énfasis será puesto en sus diversas propuestas finitistas. Además del finitismo muy radical que vimos en las notas de 1917, más estricto que el de Kronecker, hay otras opciones a las que aparece aludirse: la propia de Kronecker (identificada a veces con la aritmética recursiva primitiva), el constructivismo de Poincaré, el intuicionista, una forma que permitiría el razonamiento por inducción hasta un cierto ordinal y las diversas extensiones de la recursividad primitiva propuestas al interior mismo de la escuela de Hilbert. Las mencionamos ahora para el lector las tenga en mente y coteje con las declaraciones que aparecen en los textos que analizamos.

Con respecto a Kronocker, en varias ocasiones Hilbert lo llama "dictador", mientras que en otras lo acusa de no haber sido suficientemente radical, y adopta un finitismo mucho más extremo que el de este autor. Al final Hilbert y Bernays identificarán su finitismo con el de Kronecker. ${ }^{6}$ En las notas de cursos de 1920, encontramos los siguientes comentarios que describen la posición de Kronecker:

\begin{abstract}
En teoría de números todas las verdades son indiscutibles, las pruebas también indiscutibles e inmediatamente comprensibles al sentido común. Esto descansa en la perdurable posibilidad de comprobarlas. De hecho, las más profundas leyes de la teoría de los números pueden ser demostradas por cualquier hombre que uno encuentre en la calle [...]. [Kronecker] lucha contra cada concepto en la medida en que este hace posibles enunciados cuya corrección no es decidible en un número finito de operaciones [...]. Y Kronecker restringe la lógica también. Así como prohíbe operaciones arbitrarias con los conceptos 'reductible', 'irreductible', etc., la misma actitud mantiene ante proposiciones puramente lógicas como el tertium non datur, cuya aplicabilidad admite sólo bajo la condición de que haya la posibilidad de decidir la cuestión existencial por un procedimiento finito (citado en Ewald 1996, p. 944).
\end{abstract}

En 1922 en "La nueva fundamentación de las matemáticas", 7 Hilbert presenta su programa ya con las ideas que faltaban en 1905, si bien no todos sus componentes están completamente delimitados. Antes de presentarlo, hace una crítica de programas alternativos. No destacaremos de ello más que ciertos aspectos. Critica en conjunto a Weyl y a Brouwer a quienes equipara con Kronecker, pero las principales objeciones están específicamente dirigidas al primero. Es interesante notar aquí un contraste en el que insiste Curtis Franks (2009). Hay dos maneras contrapuestas de hacer filosofía de la matemática. Una forma es situarse desde un punto de vista filosófico supuestamente privilegiado desde el que puede descartarse como "incorrecto" cierto sector de la matemática. Ejemplos serían el intuicionismo brouweriano o el finitismo de Kronecker. El problema con esta forma de proceder es que la matemática reconstruida desde tales principios sería tan segura como éstos. Su certeza provendría de una fuente externa, normalmente una epistemología, y por lo tanto dependería de esta última. En contraste, una filosofía de las matemáticas puede respetar éstas íntegramente y obtener toda certeza posible desde el interior de la matemática misma. A veces Hilbert parece defender este punto de vista: "en las matemáticas no deben tener cabida las verdades a medias, ni tampoco pueden admitirse verdades de tipo esencialmente distinto" (Hilbert 1993, p. 37). Refiriéndose al método de Weyl dice "a partir de ese enfoque el camino hacia el análisis está vedado” (Hilbert 1993, p. 38). Hilbert insiste en la seguridad completa que tenemos en el análisis y, por tanto, parece decir que la certeza de la matemáticas es independiente de factores externos. Aunque hay otros párrafos que aparentemente contravienen esta interpretación, debemos destacar que Hilbert señala como una objeción a la filosofía de las matemáticas de Weyl el que en el marco prescrito por ella sea imposible reconstruir el análisis.

\footnotetext{
${ }^{6}$ Hilbert (1931). Hilbert \& Bernays (1934) identifican su finitismo más explícitamente con el de Skolem (1923) del que, a su vez, puede considerarse que adopta el punto de vista de Kronecker.

${ }^{7}$ Hilbert (1993), pp. 37-62.
} 
Eso muestra que la elección de un finitismo está constreñido, de una parte, por consideraciones epistemológicas y, de la otra, por la matemática misma.

Luego aparece una breve crítica a Dedekind y a Frege seguida por el párrafo siguiente que introduce la piedra angular del programa formalista:

\begin{abstract}
Más bien lo que se hace necesario como medida previa a la aplicación de inferencias y operaciones lógicas es la existencia en la representación, como algo dado, de ciertos objetos extra-lógicos discretos, intuitivamemente presentes antes de cualquier pensamiento como vivencia inmediata. Si la inferencia lógica ha de tener la seguridad que deseamos, estos objetos deben ser susceptibles de una visión global y completa de todas sus partes, y su postulación, distinción y sucesión deben presentarse ante nosotros de inmediato con los objetos mismos de manera intuitiva, como algo irreductible. En este enfoque [...] son los signos mismos los objetos de la teoría de los números. (Hilbert 1993, pp. 44-45)
\end{abstract}

Este pasaje contiene el desiderátum de la matemática finitista. Sus objetos y operaciones deben estar inmediatamente presentes y ser en todo momento "susceptibles de una visión global". Parece referirse no sólo al conocimiento directo de estos objetos, sino también al de algunas verdades a su respecto. Mancosu ha sostenido que este párrafo alude, en esta ocasión, a la intuición sensible, mientras que, fragmentos similares posteriores se refieren, más bien, a la intuición kantiana. No entraremos en ese tema.

Los siguientes pasajes en el texto que comentamos (1922) son muy importantes y los utilizaremos para aclarar las notas de 1920 en las que Sieg sitúa el nacimiento del programa, así como pasajes muy similares que aparecen en los artículos que subsiguientemente analizaremos. Hilbert dice que los objetos de la teoría de los números son los signos

$$
1,1+1,1+1+1,1+1+1+1
$$

a los que podemos llamar "números". Los últimos pueden ser respectivamente abreviados por '2', '3', ' 4 ', de tal manera que ' $2+3=3+2$ ' no es una fórmula sino un medio para comunicar que $2+3$ es el mismo signo que $3+2$. Dicho de otra forma, ' $3+2$ ' y ' $2+3$ ' son nombres (o abreviaciones) de un mismo signo y eso es lo que la ecuación comunica. Agrega que, con el objetivo de comunicarnos, usamos también letras y que ' $a+b=b+a$ ' no es una fórmula sino sólo la comunicación del hecho de que el signo numérico ' $a+b$ ' es el mismo que ' $b+a$ '. Por supuesto, no podemos tomar esta aserción en el mismo sentido que antes; ' $a+b$ ' no es el nombre del mismo signo que ' $b+a$ '. De hecho ' $a+b$ ' no es un nombre ni una abreviación, es el esquema de un nombre. Cada vez que en él coloquemos numerales concretos en lugar de 'a' y de 'b' respectivamente, obtendremos dos nombres del mismo signo y eso es lo que la ecuación comunica. Hilbert la prueba de la siguiente manera:

Hipótesis: $\mathrm{b}>\mathrm{a}$, entonces $\mathrm{b}=\mathrm{a}+\mathrm{t}$, y debemos demostrar que $\mathrm{a}+\mathrm{a}+\mathrm{t}=\mathrm{a}+\mathrm{t}+\mathrm{a}$ y, por lo tanto, que $\mathrm{a}^{+} \mathrm{t}=\mathrm{t}+\mathrm{a}$. Esto puede reiterarse hasta que los sumandos de cada lado de la ecuación coincidan (ver Hilbert 1993, p. 46).

Podría pensarse que la prueba recurre al principio del descenso infinito (o equivalentemente al principio de inducción). $\mathrm{Si}$ ' $\mathrm{a}+\mathrm{b}=\mathrm{b}+\mathrm{a}$ ' fuera una fórmula cuyos cuantificadores universales estuvieran implícitos, para su prueba requeriríamos de un axioma que asegurase que una sucesión descendente de números naturales no puede ser infinita. Hilbert lo niega. Tanto la identidad en cuestión como la prueba son meros esquemas. Para cada par de numerales concretamente dados podemos probar la conmutatividad de su suma. A nuestro parecer, a eso se refería en 1920 al decir que ' $2+3=3+2$ ' no es un caso particular de la ecuación anterior y que ésta obtiene su significado del mencionado 'procedimiento de prueba'. ¿Podemos extender esta interpretación al párrafo de 1917 que ilustra una forma de finitismo? Al parecer, no. Primeramente porque esta última interpretación será identificada con la posición de Kronecker, ${ }^{8}$ mientras que la de 1917 era, de acuerdo a Hilbert, más radical que la de este autor. En segundo lugar porque, comentando las ideas de Hilbert en 1922, Bernays dice que van a investigar si es posible fundar las presuposiciones trascendentes (es decir las que se refieren a la

\footnotetext{
${ }^{8}$ Ver más adelante.
} 
existencia de modelos de los axiomas) únicamente en el conocimiento intuitivo primitivo (ver Mancosu 1998, p. 216). Agrega la admisión de que este conocimiento incluye la inducción de contenido. En un escrito posterior (Bernays 1954) agrega que Hilbert había modificado a este respecto su posición inicial:

Originalmente Hilbert también intentó tomar el punto de vista más estrecho que no asume el concepto general intuitivo de numeral. Esto puede ser visto de su conferencia de Heidelbrg (1904). Fue ya un compromiso que él adoptara el punto de vista finitista tal y como fue presentado en sus publicaciones (Bernays 1954, p. 12, citado en Sieg 2013, p. 113).

Es decir, que Hilbert originalmente había aceptado un finitismo más radical en que la inducción de contenido no está autorizada. Aparentemente este el finitismo de 1917. Dejando de lado esta cuestión, sostendremos que los párrafos anteriores y otros más tardíos con que Hilbert ilustra el método finitario por él admitido son compatibles tanto con la interpretación Brouwer-Heyting-Kolmogorov de los cuantificadores como con las restricciones del finitismo kroneckeriano. ${ }^{9}$ Ambas nociones fueron explicadas (en el sentido carnapiano del término) algunos años más tarde y estuvo claro entonces que eran nociones distintas.

Con la distinción entre esos dos tipos de prueba de inducción, Hilbert y Bernays responden a la objeción de Poincaré: la prueba del principio de inducción completa (es decir, de su consistencia) recurrirá a la forma de inducción que podríamos llamar de contenido y de la que Bernays dice que "se relaciona sólo a algo completa y concretamente dado" y que no "usa el concepto general de número entero" ni "opera con variables de manera esencial" (Bernays 1922, en Mancosu 1998, p. 221). No habría, por tanto, ninguna circularidad. La inducción de contenido estaría basada en la intuición del signo.

Cuando Hilbert en "La nueva fundamentación de la matemática", texto de 1923, se pregunta en "si (y en qué medida) la teoría de los números puede erigirse sobre la base puramente intuitiva de los signos concretos" (Hilbert 1993, p. 45) está en el segundo de los estadios a que alude Sieg en las notas de 1920. La respuesta es que es imposible construir así la totalidad de las matemáticas:

Ya en el paso a la aritmética superior y al álgebra, por ejemplo, esto es, cuando queremos hacer afirmaciones sobre un número infinito de números o de funciones, este procedimiento concreto resulta del todo insuficiente. La razón de ello es que no podemos escribir numerales o abreviaturas para un número infinito de números. (Hilbert 1993, p. 47)

Para avanzar es necesario axiomatizar la teoría y proceder a una prueba de consistencia de la manera ya referida. En este programa la matemática concreta se traslada al nivel de la metamatemática. Las fórmulas de la teoría se convierten en lo que antes eran los numerales. Aquí se hace una demarcación estricta entre "fórmulas y demostraciones formales, por un lado, y los argumentos y demostraciones concretas por otra" (Hilbert 1993, p. 48). Estamos ya en el tercer estadio referido de las notas de 1920, el de la constitución del programa.

Enseguida presenta un breve ejemplo de cómo podría proceder una prueba de consistencia de este tipo para un sistema formal muy sencillo. Su idea es ir ampliando este sistema y probando en cada caso que el sistema resultante es consistente. La prueba mencionada recurre a lo que hemos llamado inducción de contenido. En un artículo de ese año, ${ }^{10}$ Bernays ilustra este método de prueba, con los dos siguientes ejemplos:

1. Si el signo + figura alguna vez en una prueba concretamente dada, entonces al examinar la prueba uno encuentra un lugar donde este ocurre por vez primera.

2. Si uno tiene un procedimiento general para eliminar de una prueba con cierta propiedad concretamente describible la primera figuración del signo $\mathrm{Z}$, sin que la prueba pierda la propiedad $\mathrm{E}$ en el proceso, uno puede, por aplicaciones sucesivas del proceso, remover completamente el signo Z de tal prueba, sin perder la propiedad E (Bernays 1922, en Mancosu 1998, p. 220).

\footnotetext{
9 Sieg dice que este párrafo tiene el mismo sentido (de los cuantificadores desde el punto de vista finitista) que el que aparece en el artículo de 1925, y que a su vez es el que se expone en algunos párrafos de Grundlagen der Mathematik. Agrega que es muy similar al de Weyl (Sieg 1981, p. 117).

10 “On Hilbert's Thought Concerning the Grounding of Arithmetic", en la versión inglesa de Paolo Mancosu (1998), pp. 215-222.
} 
¿En (2) también debió agregar que se trata de una prueba concretamente dada es decir, que la cláusula se refiere a un esquema de prueba? Ambos ejemplos parecerían tomados (con ligeras variaciones) de la prueba de Hilbert excepto por una salvedad importante: ésta procede por reducción al absurdo. El sistema en cuestión sólo tiene como constantes lógicas símbolos para la cuantificación universal y el condicional. Hay un símbolo para la desigualdad. Así es que Hilbert demuestra que una prueba concretamente dada de ' $a \neq a$ ' (siendo que ' $a=a$ ' es un axioma) llevaría a un absurdo en el metalenguaje. Aquí surge una diferencia importante para evaluar la respuesta dada a Poincaré. La prueba de ' $a+b=b+a$ ' era un esquema; por cada ecuación de este tipo concretamente dada, el esquema provee instrucciones para probarla. No requerimos del principio de inducción. En cada caso concreto, el número de pasos descendentes es finito. Ahora, la prueba de consistencia se presenta como algo análogo. La demostración de consistencia es supuestamente un esquema para trabajar con cada prueba concreta de contradicción; ahora bien, si el sistema es consistente, tal prueba no existe. Se trata de una proposición contrafáctica. ¿No hemos pasado con ello a otra forma de finitismo? En todo caso este tipo de reducción al absurdo es admisible para el intuicionista.

Aunque sólo nos interesa ahora el finitismo como método de prueba en el metalenguaje, algunos de los sucesivos sistemas axiomáticos que Hilbert propone en este artículo podrían estar inspirados por reflexiones acerca de lo que debe ser un sistema finitario. Por ejemplo, aún en la extensión al cálculo funcional (con variables para funciones) presentada en este artículo, no aparece ningún símbolo para la negación ni para el cuantificador existencial. En lugar de este último, Hilbert propone introducir "signos de funciones individuales por medio de una especie de definición implícita, esto es, produciendo realmente "lo que existe” (Hilbert 1993, p. 58). ¿Por qué procede de este modo? Bernays lo explica de la siguiente manera. El programa formalista retiene algo de los logros logicistas, a saber, la posibilidad de codificar el razonamiento de forma puramente mecánica y sintáctica para así convertir la prueba en objeto de estudio matemático. Por otro lado, Bernays ve el constructivismo como un ensayo de sustituir los axiomas (o presuposiciones) existenciales por postulados constructivos (como los de Euclides). Esa tendencia habría sido retomada por Kronecker y más tarde por Weyl y Brouwer y, como es de esperarse, no encuentra la aprobación de Bernays. Sin embargo, en su opinión, Hilbert retoma (a nivel matemático) algo de inspiración constructivista cuando introduce símbolos funcionales definidos implícitamente por axiomas (Bernays 1922, en Mancosu 1998, p. 219).

En una conferencia de ese mismo año, titulada "Los fundamentos lógicos de las matemáticas", publicada en 1923, Hilbert presenta de nuevo a través de ejemplos las principales ideas de su programa. Esta vez no hace ninguna crítica a sus adversarios. Introduce un formalismo para un fragmento finitista de la aritmética elemental (con axiomas que permiten la inducción de contenido, pero sin un axioma para el principio de inducción completa). Es curioso que allí aparezca como axioma una formulación del principio del tercero excluido. Sin embargo, en el contexto de ese sistema (aún sin cuantificación) es una regla finitariamente válida. Como es sabido, Tait $(1981,2002)$ ha sostenido que para Hilbert el razonamiento finitista se reduce a la aritmética recursiva primitiva. En este punto encontramos una primera indicación al respecto:

A partir de estas bases y haciendo uso de una lógica "finitista" al realizar consideraciones puramente intuitivas (entre las que hay que contar a la recursión y a la inducción inductiva para totalidades finitas), es posible obtener la teoría elemental de los números, sin que para ello se tenga que recurrir a modos de inferencia dudosos o de alguna manera problemáticos (Hilbert 1993, pp. 66-67).

Aunque la teoría de las funciones recursivas aún no había sido plenamente desarrollada, no hay duda de que algunos pasos en esa dirección había sido dados en la escuela de Hilbert, como lo muestra al final del artículo el uso de un esquema de recursión simultánea. Hay que tomar en cuenta que el célebre artículo de Skolem (1923) aún no había aparecido. Después de señalar que es posible probar finitariamente la consistencia del sistema hasta entonces presentado, Hilbert traza nítidamente la frontera que separa la aritmética de contenido de la aritmética infinitaria:

¿En qué momento se trasciende por primera vez la esfera de lo intuitivo y finito? Evidentemente cuando nos servimos de los conceptos "todo" y "existe" [...] [cuando son aplicados a totalidades infinitas]. Las 
expresiones "hay" y "se presenta, aparece" tienen obviamente el mismo significado cuando se habla de totalidades infinitas. En relación a totalidades infinitas solamente el segundo de estos conceptos tiene cierta claridad. (Hilbert 1993, pp. 67, 69-70)

Hilbert parece retomar aquí las críticas intuicionistas a la aplicación de la lógica clásica a totalidades infinitas y la compara con la aplicación descuidada de reglas que valen para sumas ordinarias a series infinitas. La cuantificación existencial con dominios infinitos equivale a una disyunción infinita que no se rige forzosamente por las mismas reglas que la disyunción ordinaria "a no ser que la teoría de la demostración lo permita)". Esta última cláusula alude a que la prueba finitaria de consistencia de un sistema validaría la lógica que este formaliza de la misma manera que (utilizando la inducción de contenido) garantizaría el uso del principio de inducción completa. Como vemos, Hilbert parece retomar al pie de la letra la crítica intuicionista al uso de los cuantificadores clásicos. Aquí se presenta el cálculo épsilon (o más bien de su dual) que había aparecido de manera incipiente desde las lecciones de 1921 a 1923. Este contiene un símbolo funcional aplicable a predicados que es introducido con un solo axioma ("de transfinitud”), a saber, $\mathrm{A}(\tau \mathrm{A}) \rightarrow \mathrm{A}(\mathrm{x})$. Es decir, ' $\tau$ ' asocia a 'A' un objeto tal que, si satisface este predicado, entonces todos los objetos también lo satisfacen. Este operador permite definir los cuantificadores clásicos y reformular el cálculo de predicados de primer orden. Hilbert esboza un método tal que, dada una fórmula, permitiría eliminar en ella las figuraciones de este operador (y con él de todas las variables) hasta obtener una fórmula decidible.

En 1924, Ackermann presentó su disertación “Begründung des 'tertium non datur' mittels der Hilbertschen Theorie der Widerspruchsfreiheit" (realizada bajo la supervisión de Bernays). En ella ofrece una prueba de consistencia para una formulación en segundo orden de la aritmética recursiva primitiva. Es interesante que para ello recurre implícitamente a la inducción transifinita hasta $\omega^{\omega^{\omega}}$. Trece años más tarde Ackermann hace a Bernays la observación siguiente: "yo no sé, por cierto, si tú estás consciente (yo en ese tiempo no lo consideré como una transgresión al punto de vista finito más estrecho), que inferencias transfinitas son usadas en mi disertación" (citado en Zach 2001, p. 85). Richard Zach comenta que Bernays "había claramente estudiado la disertación de Ackermann. No hay registro de que Bernays o Hilbert tuvieran objeciones a los métodos usados en la disertación de Ackermann. Así puede concluirse que el uso de la inducción transfinita fue considerada como aceptable desde el punto de vista finitista" (Zach 2001, p. 85). Evidentemente este tipo de prueba va más allá del constructivismo intuicionista. ¿Cómo pudo pensarse que una prueba que emplea una inducción más allá de $\omega$ es finitaria? Tal vez porque en los árboles de prueba que en ella aparecen el camino que va de cualquier nodo hacia cualquiera de los axiomas que sirven para probarlo es finito. Esto muestra que la noción de "finitismo" a la que aludían Hilbert y Bernays no estaba aún claramente delimitada.

En 1925 Hilbert dicta su conferencia "Acerca del infinito" (Hilbert 1993, pp. 83-121) que puede ser considerada como una de las exposiciones más acabadas de su programa de fundamentación. En ella aparecen mejor delineados algunos rasgos del mismo ya presentes en los dos artículos anteriores, pero también algunos elementos nuevos. Hilbert presenta su programa como análogo al trabajo de Weierstrass en la fundamentación del análisis y a la vez como una continuación del mismo. Se trata de mostrar que la alusión al infinito en matemática es sólo una forma de hablar. Esta formulación parece más apropiada al trabajo de fundamentación del análisis que al programa de Hilbert. Es verdad que en cierto sentido se trata de controlar el uso de procedimientos infinitarios por medio de restricciones y argumentos finitarios, pero tratarlo como una forma de nominalismo pudo dar pie a malentendidos. Enseguida Hilbert muestra cómo, aparentemente, el infinito sólo aparece en matemáticas y ofrece una introducción a la teoría cantoriana de los ordinales para desembocar en las paradojas. Explica entonces los elementos de su programa esta vez con una alusión aprobatoria a Kant: para la constitución o desarrollo de la matemática no basta la lógica. Ciertos objetos deben ser dados en la intuición para que la lógica pueda ser aplicada y estos objetos son las sucesiones de signos y sus concatenaciones. El kantismo de Hilbert no va más allá que esta breve alusión a la insuficiencia de la lógica y a la necesidad 
subsiguiente de un elemento "intuitivo". ${ }^{11}$ Enseguida Hilbert reitera las observaciones concernientes a las fórmulas y esquemas simbólicos que vimos en el artículo de 1922 y la misma prueba de la "conmutatividad de la adición". Es interesante cómo ahora ilustra el paso de lo intuitivo a lo infinitario. Se trata del teorema euclideano de que si p es un número primo hay otro número primo entre $\mathrm{p}^{+1}$ y $\mathrm{p} !+1$. Este es un enunciado finitario. Sin embargo, la lógica clásica autoriza a derivar de él la afirmación de que existe un número primo mayor que $\mathrm{p}$ (o que hay una infinidad de primos), el cual ya no es finitario. Concluye Hilbert que el transfinito aparece cuando un enunciado existencial no puede reducirse a una disyunción finita. En unas notas de 1920 (ver Ewald 1999, p. 944), dice que este ejemplo le fue sugerido por Kronecker. Es muy curioso porque, como vimos, a veces se refiere a él con calificaciones muy agresivas, pero en 1925 parece adoptar una posición muy similar, a saber que, desde el punto de vista finitista, no es posible negar una proposición universalmente cuantificada, ni es válido el tertium non datur. Hilbert no dice nada, en cambio, sobre los conceptos permitidos, pero evidentemente en el finitismo estos deben restringirse a la condición de que las pruebas a que dan lugar sean perfectamente perspicuas. Por supuesto el finitismo de Hilbert se limita a la metamatemática, mientras que el de Kronecker, no. Hemos visto ya su posición con respecto a los juicios existenciales. Por otro lado, un enunciado universal no es considerado como expresando una proposición universalmente cuantificada sino como "un juicio hipotético que afirma algo con tal de que dispongamos ya de un numeral" (Hilbert 1993, p. 99).

Aquí aparece otro elemento del programa de Hilbert, a saber, la idea de que el matemático extiende una teoría agregando elementos "ideales" que tienen como función redondear y simplificar la teoría original. Así, la introducción de números imaginarios simplifica las leyes del álgebra. La novedad es que Hilbert propone introducir de esta manera cierto género de enunciados: "tenemos ahora que añadir a los enunciados finitos los enunciados ideales, conservando de este modo las reglas de la lógica aristotélica en su simplicidad original" (Hilbert 1993, p. 100). La adición de enunciados ideales tiene que ser autorizada por una prueba finitaria de consistencia que los considere como meros signos desprovistos de significado y sólo regidos por reglas sintácticas. De esta manera, los métodos de Kronecker, o algunos similares, quedan restringidos a la metamatemática, que se convierte en la piedra de toque de toda teoría axiomática. ${ }^{12}$

Con respecto a la teoría de la recursividad, hay notables avances en la conferencia de 1925. Vimos cómo, en 1923, aparecía ya un esquema general de recursión simultánea. Ahora Hilbert extiende la recursión primitiva en tres sentidos: a) a diferentes tipos de variables; b) a dominios transfinitos; y c) a la recursión general. Claramente clasifica a la recursión (en los números naturales) y a la sustitución como procedimientos finitistas. Cuando se trata de la función de Ackermann, Hilbert observa que se trata de otro tipo de recursión (que no es reductible a la recursión primitiva fue demostrado por Ackermann en 1928):

pero estas recursiones no serían recursiones ordinarias, de las que van paso a paso; en su lugar llegaríamos a una variedad de recursiones simultáneas, es decir, una recursión sobre diferentes variables al mismo tiempo; y solamente podríamos transformarlas en recursiones ordinarias, paso a paso, usando la notación de variable funcional. (Hilbert 1925, en van Heijenoort 1967, p. 388)

El artículo de 1927, "The Foundations of Mathematics", ${ }^{13}$ contiene una exposición muy completa del programa formalista y, de nuevo, una aclaración de la diferencias entre el razonamiento de contenido y el formal de nuevo utilizando el ejemplo de la fórmula que expresa la conmutatividad de la adición. A pesar de su riqueza no podemos más que señalar aquí los puntos que importan a nuestro tema. El artículo comienza señalando la insuficiencia de la lógica para fundar cualquier disciplina científica. Se requieren siempre que ciertos objetos concretos sean dados en la representación. En el caso de las

\footnotetext{
${ }^{11}$ No podría decirse lo mismo de Bernays.

${ }^{12}$ Hilbert afirma que también los planteamientos del intuicionismo deben someterse a este tribunal (p. 108). Esto parecería sugerir alguna distancia con respecto a esta escuela, lo que parecería ir contra la interpretación que estamos defendiendo, pero pudiese ser que se refiriera a las ideas que Brouwer introdujo en el análisis y que ordinariamente no son aceptadas en las matemáticas clásicas.

${ }^{13}$ Ver van Heijenoort (1967), pp. 464-479.
} 
matemáticas esos objetos son los signos concretos. Llama la atención que no haya ninguna referencia a Kant. La aplicación de la lógica -dice Hilbert- requiere que ciertos "objetos concretos extra-lógicos que están intuitivamente presentes como experiencia inmediata previa a todo pensamiento" (Hilbert 1927, en van Heijenoort 1967, p. 465). Es una fórmula muy lejana del kantismo ortodoxo. Enseguida ilustra cómo en su teoría de la demostración la matemática debe ser completamente formalizada e introduce allí un sistema formal para el cálculo de predicados. Hay un axioma para el operador épsilon que permitirá definir los cuantificadores clásicos y demostrar sus propiedades usuales. Aunque en el sistema habrá variables proposicionales, el operador épsilon (y, por consiguiente, los cuantificadores) es de primer orden. Siguen axiomas (mejor dicho, esquemas de axiomas) para la igualdad y para los números naturales, el segundo de los cuales representa el principio de inducción. Sin embargo, se contempla inmediatamente una extensión a los ordinales transfinitos (con tipos de variables para cada clase de ordinales) con los axiomas de inducción correspondientes. Asimismo, menciona la introducción de nuevos conceptos matemáticos por medio de axiomas recursivos. Por recursión Hilbert se refiere a sistemas de fórmulas que definen una función explícitamente en el caso base y para un argumento indeterminado en términos de los valores de la función en argumentos menores. Es decir, se refiere a la recursión primitiva. Lo ilustra con una serie de ejemplos de funciones de números naturales.

Los párrafos que siguen son cruciales a nuestro tema. Dice allí que cuando se trata de la aritmética elemental "podemos obtener y demostrar sus verdades a través de razonamientos intuitivos de contenido. Cuando tomamos esta vía las fórmulas que allí aparecen sólo son utilizadas para la comunicación. Las letras designan cifras y una ecuación comunica el hecho de que dos cifras coinciden" (Hilbert 1993, p. 151). Este párrafo coincide puntualmente con las explicaciones que al respecto aparecen en el artículo de 1923. Las cosas cambian, dice Hilbert, cuando pasamos al álgebra:

\begin{abstract}
El álgebra sobrepasa de una manera esencial a la aritmética de contenido. Por ejemplo, la fórmula del álgebra $1+a=a+1$, donde $a$ es una verdadera variable aritmética, ha cesado de ser únicamente la comunicación de un contenido; esta fórmula es un cierto objeto formal [cuya demostración] requiere del axioma de inducción. Las fórmulas $1+3=3+1,1+7=7+1$, que son igualmente susceptibles de ser verificadas por medio de un razonamiento de contenido, sólo pueden obtenerse de la fórmula algebraica anterior por un proceso demostrativo, substituyendo formalmente $a$ por las cifras 3 y 7 , es decir, aplicando una regla de substitución. (Hilbert 1993, pp. 151-152)
\end{abstract}

Recordemos que varias veces desde 1920 insiste en que ' $x+y=y^{+} x$ ' no es una fórmula en cuya prueba se asuma el concepto general de número, ni es una aserción para todos los números de la cual ' $2+3=3+2$ ' sería un caso particular. Como vimos, allí se refiere a un esquema que sirve a la comunicación de un contenido. Hay, además, la fórmula ' $x+y=y+x$ ' con variables ' $x$ ' e ' $y$ ' que se demuestra formalmente usando el axioma de inducción (y, con ello, el concepto general de número natural). De ella derivan sus instancias particulares por medio de la regla de sustitución. Estas proposiciones formales que se demuestran siguiendo reglas sintácticas pueden ser consideradas como las entidades ideales de la teoría.

¿Por qué se requiere esta extensión (del paso 2 al 3 en términos de las notas de 1920)? Porque la aritmética de contenido no nos permite llegar muy lejos. Dice Hilbert que ' $a+1=1+a$ ' "donde a designa un número cualquiera dado" es la expresión de una comunicación "que no sería susceptible de ser negada, pues la proposición de que hay un número a tal que $a+1 \neq 1+a$ se produce no tiene ningún sentido finitista" (Hilbert 1993, p.151). Para la actitud finitista el principio del tercero excluido no es válido.

\title{
2.3. El finitismo del programa
}

¿Puede identificarse el finitismo defendido por Hilbert estos años con algún otro de los que hemos mencionado? Es difícil decirlo con precisión. Hemos sugerido que los párrafos concernientes a la conmutatividad de la adición que aparecen desde 1920 hasta 1927 son compatibles con la interpretación Brouwer-Heyting-Kolmogorov de los cuantificadores. Un enunciado universalmente 
cuantificado se prueba a través de un esquema de prueba que produce pruebas para sus ejemplificaciones concretas. Un enunciado existencial sólo tiene sentido si está acotado a un intervalo y su prueba consiste en proveer un mecanismo que permite obtener el objeto en cuestión en un tiempo prefijado. Por otro lado, Hilbert identifica en esos años la posición de Brouwer con la de Kronecker. Testimonio de ello es el siguiente párrafo del artículo de 1927: "Brouwer dice -exactamente como en otro tiempo Kronecker- que ninguna proposición de existencia tiene sentido, a menos que comporte al mismo tiempo la construcción de entidades de las que afirman la existencia" (Hilbert 1927, en van Heijenoort 1967, p. 474).

Desde luego, puede objetarse que esto no es posible porque Hilbert y Bernays pretendían responder a la crítica de Poincaré referente al principio de inducción (hasta $\omega$ ), mientras que el intuicionista acepta sin reservas esta forma de razonamiento. Pero recordemos que la inducción intuicionista tiene como conclusión un enunciado universalmente cuantificado y ese cuantificador es el intuicionista. Su comportamiento frente a la negación lo distingue de su contraparte clásica. En ese sentido, Hilbert y Bernays creyeron responder a Poincaré. Por otro lado, vimos que también aceptaron en el ámbito finitista pruebas como la de Ackermann que, por supuesto, exceden las restricciones intuicionistas. Al respecto creemos que Hilbert y Bernays no parecían haber decidido claramente qué noción tenían en mente y es claro, además, que tenían una dificultad para distinguir conceptos y métodos que su trabajo mismo contribuyó a delimitar.

Asimismo podría oponerse a nuestra interpretación el que Hilbert realiza críticas a Brouwer particularmente acervas en el artículo de 1927, año en el cual la rivalidad entre ambos autores llegó a un nivel personal. Sin embargo, las objeciones a Brouwer aluden a la amputación que la matemática tendría que sufrir para satisfacer las demandas intuicionistas. Es decir, van contra la imposición de esas restricciones en el nivel matemático, no en el metamatemático. Se dirigen a quien se hubiese quedado en el segundo estadio de las notas de 1920.

Sin embargo, la identificación del finitismo metamatemático del programa formalismo con el intuicionismo no significa que Hilbert haya aceptado en lo más mínimo la filosofía con que Brouwer justifica su programa y que comprendía la intuición de la temporalidad a la base de la aritmética. La intuición del signo es algo muy diferente y conserva sólo una vaga reminiscencia del filósofo de Königsberg. Mientras que para Brouwer la filosofía pone límites a la matemática con sentido, Hilbert se mueve entre unos pocos principios epistemológicos y el deseo de conservar la matemática clásica. Como vimos, una crítica a Weyl es que su epistemología no le permite reconstruir el análisis clásico. Podríamos suponer que, para Hilbert, el conocimiento matemático es un hecho y es una piedra de toque de una buena epistemología. Al final del artículo de 1927, dice que "la matemática es una ciencia sin presupuestos" (Hilbert 1927, en van Heijenoort 167, p. 479) y con ello distingue su posición de la de sus rivales (que descansan en una filosofía o en presupuestos matemáticos). Eso supone que el programa podría haber satisfecho ambos requerimientos, en cuyo caso el sustrato de que parte sería evidentemente verdadero y no requeriría de una epistemología que explicara esa evidencia.

Podría preguntarse por qué si la metodología finitista de Hilbert (para la metamatemática) coincidía con la de Brouwer (a nivel matemático), no hay declaraciones explícitas al respecto. La respuesta es que sí hay algunas esparcidas en los textos, si bien no todas van en el mismo sentido.

Mancosu (2010, p. 144) menciona una carta de Bernays a Hilbert (del 25 de octubre de 1925) en donde Bernays dice haber notado una diferencia entre intuicionismo y finitismo, lo cual supone, por supuesto, que había una tácita identificación entre ambas metodologías. En 1927 von Neumann escribió que "la prueba intuitiva (de contenido) [de la consistencia] debe proceder en el sentido de la lógica intuicionista de Weyl y Brouwer". ${ }^{14}$ Es interesante que enseguida agrega que con ello se busca reducir al absurdo al intuicionismo. Eso parece implicar que Hilbert adopta la metodología intuicionista y la identifica con el finitismo como parte de una estrategia dialéctica y no necesariamente porque esté convencido de las virtudes epistémicas del intuicionismo. Sin embargo, esa identificación

${ }^{14}$ Citado en Mancosu (2010), p. 144. 
no parecía fuera de lugar. Bernays la afirma implícitamente en $1930^{15}$. Para Mancosu esto representa una ampliación del punto de vista original que sólo ocurrió hasta finales de los años veintes (Mancosu 2010, p. 144). Nosotros pensamos que sucedió al menos desde 1922. Por otro lado, en 1931 Hilbert identifica plenamente su finitismo con la posición de Kronecker: "Kronecker claramente expresó una concepción que ilustró con numerosos ejemplos; esta concepción coincide hoy esencialmente con nuestro modo finito de pensamiento" (Hilbert 1931, en Mancosu 1998, p. 267).

La sucesiva clarificación de las nociones en juego por medio de la constitución de la teoría de las funciones recursivas, de la demostración de los teoremas de incompletud, así como de la evolución de la teoría de la prueba, permitieron a Hilbert y a Bernays determinar con más precisión el finitismo de su programa. Por ejemplo, en 1933 Gödel probó por medio de su "traducción negativa" que la aritmética clásica es interpretable en la aritmética intuicionista (lo cual muestra que ésta no puede ser finitista). En las primeras secciones de Grundlagen der Mathematik, Hilbert y Bernays caracterizaron de manera informal, pero detallada, el punto de vista finitista en teoría de números. Constatando que desde esta perspectiva el tercero excluido no es válido se refieren aprobatoriamente a Brouwer. Sin embargo, más adelante califican al intuicionismo como una extensión del punto de vista finitista

en la medida en que Brouwer admite que una hipótesis sea introducida en la presencia de una conclusión (o una prueba), sin que la conclusión (o la prueba) sea determinada por una condición intuitiva. Así, por ejemplo, desde el punto de vista de Brouwer, se admite que una proposición de la forma "si bajo la hipótesis A la proposición B vale, entonces C vale también", o "la hipótesis de que A es refutable conduce a una contradicción" [...] según la manera de expresarse de Brouwer: "el absurdo del absurdo”. (Hilbert \& Bernays 1934-1939, p. 43) ${ }^{16}$

Por otro lado, el punto de vista finistista (en la aritmética) es identificado con la posición de Kronecker. ${ }^{17}$

\section{Conclusiones}

Hemos mostrado cómo algunos elementos del programa formalista estaban ya presentes alrededor de 1900 en las propuestas de Hilbert para fundamentar las matemáticas. Nos referimos al estructuralismo, a la preferencia por el método axiomático, el logicismo y a la necesidad de probar consistencia de teorías axiomáticas. Analizamos brevemente sus diferencias con Frege en lo referente al uso del método axiomático para hacer resaltar su propia propuesta al respecto. Vimos que, aunque hay una cierta concepción estructural en su pensamiento, ninguna de las formas específicas que identifica Shapiro puede serle claramente atribuida. Analizamos en qué sentido puede atribuírsele a Hilbert una defensa del logicismo a principios del siglo XX, cómo a veces defiende esta postura y a veces se distancia de ella, para terminar alejándose de ella definitivamente. Sin embargo, algún componente del logicismo sobrevivió en la forma madura del programa bajo la idea de que la formalización de la lógica es, en algún sentido, completa o suficiente. En la segunda parte, vimos cómo Hilbert tomó alguna vez el finitismo como una posible base para dicha fundamentación, antes del nacimiento de su programa en 1920. Analizando los ejemplos de metodología finitista dados por Hilbert en la década de los veintes concluimos que son compatibles con la interpretación Brouwer-Heyting-Kolmogorov, sin que eso implique de su parte la adopción de la perspectiva filosófica de Brouwer. A pesar de eso, Hilbert parece a veces oscilar hacia otras formas de finitismo. Esto se debió a su extraordinario afán por explorar otras vías y al hecho de que los conceptos aún no estaban completamente delimitados en ese periodo. Hilbert y su escuela contribuyeron en gran medida en darle un impulso al finitismo e investigar sus ramificaciones y consecuencias.

\footnotetext{
${ }^{15}$ Ver penúltima nota.

${ }^{16}$ La misma observación, formulada de otra manera, aparece en una apéndice que Bernays agregó en 1976, a un largo artículo en que comenta el programa de Hilbert. Esa nota se presenta como una corrección al punto de vista sostenido en el artículo original, lo cual supone que Bernays identificaba intuicionismo y finitismo.

${ }^{17}$ Ver, por ejemplo, Hillbert \& Bernays (1934-1939), p. 42.
} 
Ackermann, W. (1924), “Begründung des 'tertium non datur' mittels der Hilbertschen Theorie der Widerspruchsfreiheit”, Tesis doctoral, Universität Göttingen.

Benis-Sinaceur, H. (2015), “Is Dedekind a Logicist? Why Does Such a Question Arise?”, en Benis-Sinaceur, H., Panza M. y G. Sandu (eds.), Functions and Generality of Logic, Reflections on Dedekind's and Frege's Logicism, Berlin: Springer, 1-57.

Bernays, P. (1922), "Über Hilberts Gedanken zur Grundlegung der Arithmetik", Jahresbericht der Deutschen Mathematikervereinigung 31: 10-19. (Versión inglesa de Paolo Mancosu: "On Hilbert's Thoughts Concerning the Grounding of Arithmetic", en Mancosu (1998), pp. 215-233.)

Bernays, P. (1954), "Zur Beurteilung der situation in der beweistheoretischen Forschung”, Revue Intenationale de Philosophie 8: 9-13. Discusión: 15-21.

Bernays, P. (2003), Philosophie des Mathématiques, Paris: Vrin.

Dedekind, R. (1888), Was sind und was sollen die Zahlen, Braunschweig: Vieweg.

Dedekind, R. y H. Benis-Sinaceur (2008), La Création des Nombres, Paris: Vrin.

Detlefsen, M. (1986), Hilbert's Program: An Essay on Mathematical Instrumentalism, Berlin: Springer.

Ewald, W. (1999), From Kant to Hilbert, Vol. 2, Oxford: Oxford University Press.

Ferreirós, J. (2009). "Hilbert, Logicism, and Mathematical Existence”, Synthese 170(1): 33-70.

Ferreirós, J. (2011), “On Dedekind Logicism”, http: philsci-archive.pitt.edu/13075.

Franks, C. (2009), The Autonomy of Mathematical Knowledge, Hilbert's Program Revisited, Cambridge: Cambridge University Press.

Frege, G. (1953), The Foundations of Arithmetic, New York: Harper.

Frege, G. (1980), Philosophical and Mathematical Correspondence, Chicago: The University of Chicago Press.

Hilbert, D. (1905), "Über die Grundlagen der Logik und der Arithmetik", en Verhandlungen des Dritten Internationalen Mathematiker-Kongresses in Heidelberg vom 8. bis 13. August 1904, Leipzig: Teubner, 1905, pp. 174-185. (Versión inglesa: "On the Foundations of Logic and Arithmetic", en van Heijenoort (1967), pp. 145-56.)

Hilbert, D. (1925), “Über das Unendliche”, Mathematische Annalen 95: 161-190. (Versión inglesa: “On the Infinite”, en van Heijenoort (1967), pp. 367-392.)

Hilbert, D. (1927), "Die Grundlagen der Mathematik”, Abhandlungen aus dem mathematischen Seminar der Hamburgischen Universität 6 (1928): 65-85. (Versión inglesa: “On the Foundations of Logic and Arithmetic”, en van Heijenoort (1967), pp. 464-479.)

Hilbert, D. (1929), "Probleme der Grundlegung der Mathematik”, Mathematische Annalen 102: 1-9.

Hilbert, D. (1931), “Die Grundlegung der elementaren Zahlenlehre”, Mathematische Annalen 104: 485-494. (Versión inglesa: "The Grounding of Elementary Number Theory", en Mancosu (1998), pp. 266-273.)

Hilbert, D. (1993), Fundamentos de las Matemáticas, México: Facultad de Ciencias-UNAM.

Hilbert, D. (1999), The Foundations of Geometry, LaSalle, IL: The Open Court Publishing Co.

Hilbert, D. y P. Bernays (1934-1939), Grundlagen der Mathematik, Vols. I-II, Berlin: Springer. (Versión francesa: Fondements des Mathématiques, Paris: L'Harmattan, 2001.)

Hilbert, D. y W. Ackermann (1928), Grundzüge der theoretischen Logik, Berlin: Springer.

Largeault, J. (1992), Intuitinionisme et Théorie de la Démonstration, Paris: Vrin.

Mancosu, P. (ed.) (1998), From Brouwer to Hilbert, The Debate on the Foundations of Mathematics in the 1920's, Oxford: Oxford University Press.

Mancosu, P. (2010), The Adventure of Reason, Interplay between Philosophy of Mathematics and Mathematical Logic, 1900. 1940, Oxford: Oxford University Press. 
Poincaré, H. (1905-1906), "Les mathématiques et la logique”, Revue de Métaphysique et de Morale 13 (noviembre 1905): 815-35; 14 (enero 1906): 17-34.

Poincaré, H. (1906), “Les mathématiques et la logique”, Revue de Métaphysique et de Morale 14 (mayo 1906): 294-317.

Shapiro, S. (1997), Philosophy of Mathematics, Structure and Ontology, Oxford: Oxford University Press.

Sieg, W. (2013), Hilbert's Programs and Beyond, Oxford: Oxford University Press.

Skolem, T. (1923),"The Foundations of Elementary Arithmetic established by Means of the Recursive Mode of Thought, Without the Use of Apparent Variables Ranging over Infinite Domains”, en van Heijenoort (1967), pp. $302-333$.

Stenlund, S. (2010), “Different Senses of Finitude: An Inquiry into Hilbert's Finitism”, Synthese 185: 335-363.

Tait, W. (1981), "Finitism”, Journal of Philosophy 78: 524-546.

Tait, W. (2002), "Remarks on Finitism”, en Sieg, W., Sommer, R. y C. Talcott (eds.), Reflections on the Foundations of Mathematics: Essays in Honor of Solomon Feferman, Lecture Notes in Logic, Vol. 15, Urbana: Association for Symbolic Logic, pp. 407-416.

van Heijenoort, J. (1967), From Frege to Gödel, A Source Book in Mathematical Logic, 1879-1931, Cambridge, MA: Harvard University Press.

Zach, R. (2001), Hilbert's Finitism, Historical, Philosophical and Mathematical Perspectives, Tesis doctoral, University of California. 